\title{
PodCastle: A Web 2.0 Approach to Speech Recognition Research
}

\author{
Masataka Goto, Jun Ogata, and Kouichirou Eto \\ National Institute of Advanced Industrial Science and Technology (AIST) \\ 1-1-1 Umezono, Tsukuba, Ibaraki 305-8568, JAPAN
}

\begin{abstract}
In this paper, we describe a public web service, "PodCastle", that provides full-text searching of Japanese podcasts on the basis of automatic speech recognition. This is an instance of our research approach, "Speech Recognition Research 2.0", which is aimed at providing users with a web service based on Web 2.0 so that they can experience state-of-the-art speech recognition performance, and at promoting speech recognition technologies in cooperation with anonymous users. PodCastle enables users to find podcasts that include a search term, read full texts of their recognition results, and easily correct recognition errors. The results of the error correction can then be used to improve the performance of both full-text search and speech recognition. Although we know of no state-of-the-art speech recognizer that can successfully transcribe all of the various kinds of podcasts, the mechanism we propose will gradually increase the usefulness and applicability of PodCastle.
\end{abstract}

Index Terms: information retrieval, speech recognition, error correction, wisdom of crowds, Web 2.0

\section{Introduction}

Speech recognition researchers understand what sort of speech is easily recognized by speech recognizers and realize that speech recognizers perform best when dealing with clean speech. On the other hand, most end users of speech recognizers judge the effectiveness of speech recognition from their limited experiences and do not necessarily understand how useful stateof-the-art recognizers can be. Users sometimes do not adequately comprehend what sort of voices or recording conditions make recognition difficult. If they have previously had difficulty being understood by speech recognizers, they often doubt the usefulness of speech recognition and may stop using it.

The first aim of this study is to address this problem by promoting the popularization and use of speech recognition by raising end user awareness of state-of-the-art speech recognition performance. For this purpose, we launched a web service called "PodCastle" that allows a number of anonymous users to search and read podcasts, and to share the full text of speech recognition results for podcasts. Podcasts are audio programs distributed on the web, like radio shows or audio blogs. They are becoming increasingly popular because updated podcasts (MP3 audio files) can be easily and frequently downloaded by using RSS syndication feeds. Since various contents have already been published as podcasts, users can grasp the current state of speech recognition technology just by seeing the results of speech recognition applied to published podcasts. This is important because when some users experience recognition errors while speaking into a microphone, they may become uncomfortable or frustrated and lose their motivation. Such problems do not occur for PodCastle because users do not have to provide their own speech input at all.

However, even state-of-the-art speech recognizers cannot correctly transcribe all podcasts, because their contents and recording environments vary very widely. A typical approach to deal with speech contents that cannot be properly recognized is to create a speech corpus including such contents and prepare correct transcriptions to train speech recognizers. This approach, however, is impractical for PodCastle because advance preparation of a corpus covering all podcast contents will be too costly and time consuming.

The second aim of this study is to dispense with the idea of using a pre-prepared corpus to address this problem, and instead employ the efforts of a large number of users to improve speech recognition and full-text search performance. Even if a state-ofthe-art speech recognizer is used to recognize podcasts on the web, a number of errors will naturally occur. PodCastle therefore encourages users to cooperate by correcting these errors so that those podcasts can be searched more reliably. Furthermore, using the resulting corrections to train the speech recognizer, it implements a mechanism whereby the speech recognition performance is gradually improved. This approach can be described as "collaborative training for speech recognition".

In this paper, we introduce the term "Speech Recognition Research 2.0" to refer to the research approach where the current state of speech recognition technology is intentionally disclosed to users so that speech recognition performance can be improved through cooperative participation by users. This term was chosen to reflect the concept of Web 2.0 [1], since this approach brings the benefits of Web 2.0 to speech recognition research. In Section 2 of this paper, we discuss the research approach that Speech Recognition Research 2.0 represents, and in Section 3 we describe the PodCastle web service as an instance of this approach. In Section 4, we summarize the contributions of this research.

\section{Speech Recognition Research $\mathbf{2 . 0}$}

"Speech Recognition Research 2.0" is a new research approach to speech recognition which aims at improving speech recognition performance and the usage rate while benefiting from the cooperation of a number of anonymous end users. To achieve this, we propose setting into motion a positive spiral as explained in Figure 1. In the past, this spiral has not necessarily taken hold because of inhibiting factors in each of the three steps important for popularizing speech recognition. The problems affecting each of these steps are as follows:

- With regard to (i) understanding speech recognition performance, users have tended to see the results of speech recognition applied only to their own voices. Once users experience recognition problems with their voices, they tend to incorrectly assume that other people's voices will also not be well recognized. On the other hand, speech recognition researchers have a better understanding of recognition capabilities because they have more opportunity to see the results of speech recognition applied not only to their own voices but to a large speech corpus.

- As for (ii) contributing to improved speech recognition performance, users of speech dictation systems can make speech recognizers adapt to their voices by reading out pre- 
(i) Allowing users to experience speech recognition lets them better understand its performance.

(ii) Users contribute to improved speech recognition performance.

(iii) Improved performance leads to a better user experience.

Figure 1: A positive spiral leading towards greater use of speech recognition (through repetition of steps (i), (ii), and (iii)).

defined sentences or add out-of-vocabulary words. ${ }^{1}$ However, such in-house performance improvements made by each end user are not made available for re-use by other users. Only speech recognition researchers have been able to improve the performance of speech recognition as a whole. Consequently, it has been difficult to motivate a number of users to contribute to collaborative efforts that will improve performance.

- With regard to (iii) a better user experience, users have had little opportunity to experience the better performance that results from ongoing improvements made by researchers. For example, even if speech recognizers are made available as open source software (e.g., [2]), these are mainly aimed at developers; end users have little opportunity to use them directly. Also, users of most speech recognition products have only experienced performance improvements through infrequent software updates.

By addressing these problems, Speech Recognition Research 2.0 aims to change the usage of speech recognition by setting the positive spiral of Figure 1 into motion. Table $1 \mathrm{com}-$ pares this approach with the conventional approach to speech recognition research, which we will call "Speech Recognition Research 1.0". We are not suggesting, though, that Speech Recognition Research 1.0 is inferior or obsolete, and there is no doubt that continued research using the Speech Recognition Research 1.0 approach is needed. We ourselves have continued our work on Speech Recognition Research 1.0 as the foundation for 2.0. It should also be stressed that we are discussing research approaches, and not speech recognition techniques or algorithms themselves, which is why we use the term "Speech Recognition Research 2.0" instead of "Speech Recognition 2.0".

In the following, we discuss how the beneficial spiral of Figure 1 can be put into effect while also explaining the points in Table 1.

- Instead of developing stand-alone applications such as dictation or spoken dialogue by preparing a corpus for speech recognizers, Speech Recognition Research 2.0 provides a web service that allows users to search and browse opento-the-public web-based speech data such as podcasts. In this way, it promotes understanding of speech recognition performance (step (i) in Figure 1).

- When recognizing web-based speech data, however, we cannot limit the range of topics and prepare in advance a suitable corpus with its transcription. This causes many recognition errors, so Speech Recognition Research 2.0 gets users to correct the errors, thereby enabling recognition of a wide variety of speech data on an unlimited range of topics. That is, users cooperate in the preparation of full-text transcriptions as a form of annotation that can be used when

${ }^{1}$ Some systems can adapt automatically to a speaker's voice during use without the user's awareness, and some systems can automatically acquire out-of-vocabulary words. However, in neither of these cases it is possible to share this information between users.
Table 1: A comparison of the conventional approach to speech recognition research (Speech Recognition Research 1.0) and the proposed approach (Speech Recognition Research 2.0).

\begin{tabular}{c|c}
\hline \hline Speech Recognition Research 1.0 & Speech Recognition Research 2.0 \\
\hline Stand-alone application & Web service \\
Dictation & Searching/browsing \\
Corpus & Web-based data \\
Limited topics & Unlimited topics \\
Transcription & Annotation \\
Out-of-vocabulary words & Not-yet-annotated words \\
Specialist participation & User participation \\
Individual correction & Social correction \\
Personal wisdom & Wisdom of crowds \\
Completed version & Perpetual beta \\
\hline
\end{tabular}

The above table is influenced by the comparison of Web 1.0 and Web 2.0 by O'Reilly [1]. Research projects that feature more points on the right side of the table are more worthy of the name "Speech Recognition Research 2.0". However, as with Web 2.0, this does not mean that all these points have to be featured in any one project.

searching for speech data. It is important that user corrections are also used for training speech recognizers so that not-yet-corrected errors in other parts or other speech data can be reduced. ${ }^{2}$ In Speech Recognition Research 2.0, outof-vocabulary words are regarded as being nothing more than not-yet-annotated words which will be annotated (corrected) by users and then automatically added to the system vocabulary. In this way, users can contribute to improved performance (step (ii) in Figure 1).

- Furthermore, instead of confining this to individual corrections, we propose extending this user participation framework to provide a social correction framework, where a number of anonymous users can improve the performance by sharing their correction results over a web service. In this social framework, users gain a real sense of contributing to the convenience of other users, and can be motivated to contribute by seeing the correction activities made by other users. In this way, we can use the wisdom of crowds to achieve a better user experience (step (iii) in Figure 1).

In other words, Speech Recognition Research 2.0 can be described as an approach whereby a web service based on speech recognition that is permanently in beta version (perpetual beta) is launched and then improved by inviting users to use it on the web, thereby advancing the research.

As the first instance of Speech Recognition Research 2.0, we initiated the PodCastle project in January 2006. In this project, our goal is to set the positive spiral of Figure 1 into motion by providing the PodCastle web service which is based on the concepts of both Web 2.0 and Speech Recognition Research 2.0 .

\section{PodCastle: A podcast search service based on speech recognition}

PodCastle is a social annotation web service where users can search, read, and annotate podcasts in text form. Each podcast consists of a series of episodes of audio data (MP3 files) and their metadata (RSS syndication feed) that promotes its circulation. The creator of a podcast (the podcaster) can add new

\footnotetext{
${ }^{2}$ This is an original benefit of Speech Recognition Research 2.0 that is not provided by Web 2.0. For example, in other services such as Wikipedia [3] based on the wisdom of crowds, the users' contributions are limited to the articles they edit. There is no automatic improvement of other articles.
} 


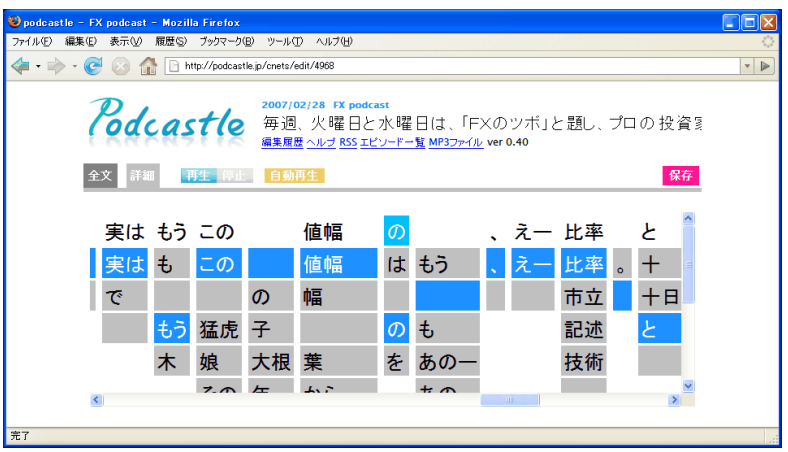

Figure 2: PodCastle screen snapshot of an interface for correcting speech recognition errors (competitive candidates are presented underneath the normal recognition results). Five errors in this excerpt were corrected by selecting from the candidates. The corrected Japanese sentence means "... well, actually the ratio of this price range and..."

episodes at arbitrary intervals (daily, weekly, etc.). With RSS, updated episodes are automatically downloaded from the web and can be stored in any type of player. Podcasts are often referred to as audio blogs and their popularity has grown because anybody can publish and download audio data with ease. Just as full-text search services are essential for accessing text web pages, there is a growing need for full-text speech retrieval services such as PodCastle.

Although there have been previous research projects for speech retrieval $[4,5,6,7,8]$, most do not provide public web services for podcasts. There are two major exceptions, Podscope [9] and PodZinger [10], which started web services for speech retrieval targeting English-language podcasts in 2005. These services use speech recognition to turn podcasts into text, and can display a list of podcasts that include a search term. In Podscope, users are shown none of the speech recognition results and only the title list is provided, although speech data around the found search term can be played back. In PodZinger, users are shown text excerpts (recognition results) surrounding the search term, allowing users to grasp the context of the podcast more easily. In contrast, PodCastle is the first service to provide full-text searching of Japanese-language podcasts. Even if the other services can also support Japanese podcasts in the future, PodCastle differs significantly in three ways:

1. Although speech recognition has been used in earlier services, they have only displayed parts of the resulting text, making it impossible to visually ascertain the detailed contents of the podcast without actually listening to it.

2. The full-text results of speech recognition have been hidden inside, so it has not been possible to search them using other existing text-based search engines.

3. Even if users find that search results are degraded by unavoidable speech recognition errors, the users have had no means of correcting these errors.

In contrast, PodCastle allows full-text results of speech recognition to be accessed by both users and external search services, and allows a number of users to cooperate with each other to improve the speech recognition performance.

\subsection{Three functions of PodCastle}

PodCastle supports three functions - searching, reading, and annotating — to satisfy all the points of Speech Recognition Research 2.0 listed in Table 1 and set the positive spiral of Figure 1 into motion. Specifically, the searching and reading functions let users better understand the speech recognition performance regarding podcasts (step (i) in Figure 1), and the annotating (error correction) function allows them to contribute to improved performance (step (ii)). This improved performance can then lead to a better user experience of searching and reading podcasts (step (iii)).

\subsubsection{Searching function}

This is a function that allows a full-text search of the speech recognition results (and the results corrected by users). When the user types in a search term, as with an ordinary text-based search engine, a list of episodes containing this term is displayed together with text excerpts around the term. These excerpts can be played back individually. By selecting one of these search results, the user is then able to access its full text by switching over to the reading function.

\subsubsection{Reading function}

With this function, as well as listening to a podcast the user can also view the text of the podcast. This allows users to understand the contents of a podcast even when audio playback is not possible, and allows them to quickly decide whether they are interested in the podcast's contents without having to listen to it. To make errors easy to discover, each word is colored according to the degree of reliability estimated during speech recognition. Furthermore, a cursor moves across the text in synchronization with the audio playback.

Because the full-text result of speech recognition being applied to each episode becomes available to external full-text search engines, such results can be discovered together with ordinary web pages by these engines. This increases the value of podcasts by bringing more users into contact with them. Since this benefits the podcasters, it will motivate them, together with other volunteer users, to use the annotating function.

\subsubsection{Annotating function}

This function allows users to add "annotations" to correct any recognition errors they may come across while searching or reading. Here, annotation means transcribing the podcast contents, either by selecting the correct candidate from the list of competitive candidates, or by typing in the correct text. For this purpose, we provide an efficient error correction interface we earlier proposed [11]. In this interface, shown in Figure 2, a recognition result excerpt is shown around the cursor and scrolled in synchronization with the audio playback. Each word in the excerpt is accompanied by other word candidates, which are generated beforehand by using a confusion network ${ }^{3}$ [12] that can condense a huge internal word graph of a large vocabulary continuous speech recognition (LVCSR) system. Note that users are not expected to correct all the errors, but can be expected to correct some errors according to their interests.

\subsection{Implementation and public release of PodCastle}

The implementation overview of PodCastle is shown in Figure 3. The web crawler collects podcasts, which can be added by users, and records them in the database manager. Those podcasts are then recognized by multiple speech recognizers one after another. When a request from a speech recognizer is received by the speech recognition manager, the next

\footnotetext{
${ }^{3}$ The confusion network was originally introduced in the context of word error minimization, which minimizes the word error rate of recognition results rather than the sentence error rate [12]. Our original idea is to apply such an efficient intermediate recognition result to generate competitive candidates for efficient error correction [11].
} 


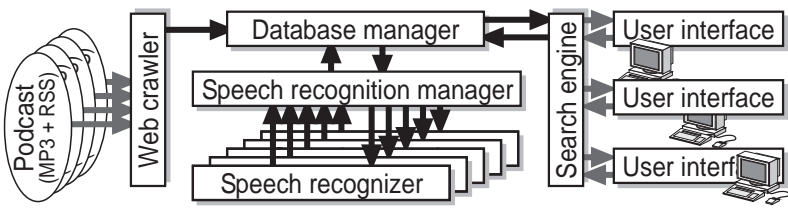

Figure 3: Implementation overview of PodCastle.

available episode to be recognized is handed over. After the recognizer finishes processing its episode, the recognition result is passed to the database manager via the speech recognition manager. The database manager controls the processing state of the podcasts and indexes their speech recognition results together with the corrections provided by users. Finally, the search engine works as a website that provides the PodCastle user interface with the three functions.

The web server of PodCastle was implemented by using a web application framework Ruby on Rails 1.1.4, a programming language Ruby 1.8.4, a web server WEBrick 1.3.1, a database MySQL 4.1.21, and a morphological parser for the Japanese language ChaSen 2.3.3. The client interface was implemented by using a scripting language JavaScript 1.5 and its library MochiKit 1.3.1, multimedia frameworks QuickTime 7 and Flash 7, and an ActionScript 2 compiler MTASC 1.12.

To recognize podcasts, audio data is first segmented into three categories - speech, music without speech, and other background sounds - by applying GMMs. Speech segments are then recognized by using our in-house LVCSR system based on an efficient N-best search algorithm [13] to generate the confusion networks. This system uses cross-word tied-state triphone HMMs trained for 39-dimensional MFCC-based features, and a $159 \mathrm{k}$-word trigram language model trained by using both large standard speech corpora and daily-updated web news. The automatic performance improvements through correction by users can be achieved through various techniques, such as those for training an acoustic model by using corrected transcriptions, for making a language model adapt to different topics by using RSS metadata and corrected transcriptions, and for registering out-of-vocabulary words by using a phonetic typewriter to estimate their pronunciation. The details of our speech recognizer are described in another paper [14].

PodCastle was released to the public at http://podcastle.jp on December 1st, 2006, and has received 7493 search requests in the three and half months since, even though we have not promoted it to the public (although we plan to soon). 124 podcasts have been registered, consisting of 5398 episodes in total. Of these, 239 episodes $(4.4 \%)$ have been at least partially corrected. We found that there are users who voluntarily cooperate in the correction as there are in other Web 2.0 services, and that podcasts recorded by famous artists and TV personalities tend to receive many corrections. One Web 2.0 principle is to trust users and we also trust users with respect to the quality of correction: in practice, the correction results obtained so far have been of high quality. Even if some users deliberately make inappropriate corrections (vandalism), though, we can develop countermeasures to acoustically evaluate the reliability of corrections. For example, we can use the likelihood of HMMs for forced alignment with the corrections.

\section{Conclusion}

We have proposed "Speech Recognition Research 2.0" as a new approach to speech recognition research that complements existing approaches. As the first instance of this approach's application, we have launched the PodCastle web service which pro- vides a search engine for podcasts on the basis of the wisdom of crowds. The technical contribution of this study is to investigate how far the performance of speech recognition and fulltext search can be improved by getting speech recognition errors corrected through the cooperative efforts of many end users. At the same time, it makes a social contribution in that it helps web users by providing the world's first public web service for full-text search of Japanese-language podcasts.

Another contribution of this study is that it demonstrates how speech recognition can be put to use in situations where a speech corpus is almost impossible to prepare in advance. Although speech recognition usually requires a sufficient corpus to provide useful results, such corpora tend to be costly and labor-intensive, thus limiting applications. On the other hand, this study has aimed at "collaborative training for speech recognition" where full-text transcriptions containing recognition errors are first disclosed and then corrected by anonymous users. Since there are many errors, we run the risk of attracting criticism, but we believe that sharing these results with users will promote further popularization and use of speech recognition. We hope that this study will prove the importance and potential of incorporating user contributions into speech recognition, and that various other projects that follow the Speech Recognition Research 2.0 approach will be done, thus adding a new dimension to this field of research.

Acknowledgments: We thank Ryutaro Kamitsu (Brazil Inc.), Shunichi Arai (Mellowtone Inc.), and Youhei Sawada for their web service implementation. We also thank the anonymous users of PodCastle for correcting speech recognition errors.

\section{References}

[1] T. O'Reilly, "What is Web 2.0 - design patterns and business models for the next generation of software," http:/l www.oreillynet.com/pub/a/oreilly/tim/news/2005/09/30/what-isweb-20.html.

[2] A. Lee, T. Kawahara, and K. Shikano, "Julius - an open source real-time large vocabulary recognition engine," in Proc. of Eurospeech 2001, 2001, pp. 1691-1694.

[3] Wikipedia, http://www.wikipedia.org/.

[4] S. Whittaker, et al., "SCAN: Designing and evaluating user interfaces to support retrieval from speech archives," in Proc. of ACM SIGIR Conf. on Research and Development in Information Retrieval, 1999, pp. 26-33.

[5] J.-M. V. Thong, et al., "Speechbot: An experimental speech-based search engine for multimedia content on the web," IEEE Transactions on Multimedia, vol. 4, no. 1, pp. 88-96, 2002.

[6] L. shan Lee and B. Chen, "Spoken document understanding and organization," IEEE Signal Processing Magazine, vol. 22, no. 5, pp. 42-60, 2005.

[7] Cambridge Multimedia Document Retrieval Project, http://mi.eng.cam.ac.uk/research/projects/mdr/.

[8] CMU Informedia Digital Video Library Project, http://www.informedia.cs.cmu.edu/.

[9] Podscope, http://www.podscope.com/.

[10] PodZinger, http://www.podzinger.com/.

[11] J. Ogata and M. Goto, "Speech Repair: Quick error correction just by using selection operation for speech input interfaces," in Proc. of Eurospeech 2005, 2005, pp. 133-136.

[12] L. Mangu, E. Brill, and A. Stolcke, "Finding consensus in speech recognition: Word error minimization and other applications of confusion networks," Computer Speech and Language, vol. 14 no. 4, pp. 373-400, 2000.

[13] J. Ogata and Y. Ariki, "An efficient lexical tree search for large vocabulary continuous speech recognition," in Proc. of ICSLP 2000 , 2000, pp. 967-970.

[14] J. Ogata, M. Goto, and K. Eto, "Automatic transcription for a Web 2.0 service to search podcasts," in Proc. of Interspeech 2007, 2007. 